\title{
توجمة رواية Di Bawah Lindungan Ka'bah ؛ مشكلات وحلول
}

\section{Rojja Pebrian}

Universitas Islam Riau, Indonesia

rojjapebrian@fis.uir.ac.id

\section{ملخص البحث}

إن الترجمـة وسيلة لنقل العلوم والمعارف مـ وإلى اللغات المختلفـة لتبـادل الثقافة والحضـارة، وبهـا يتم التعرف على ثقافة الآخر وأدبه وفنونه وإشراك الآخرين في متعة التعرف عليها. ويسعى هذا البحث إلى التعرف على بعض مشكلات ترجمة

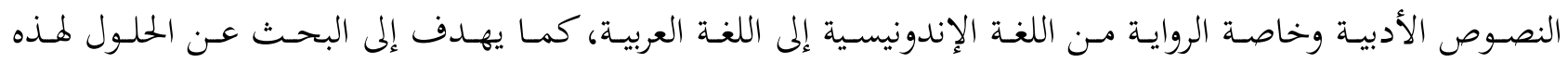
المشكلات. واختار الباحث لعمل الترجمة إحدى أعمال حمكا الأدبية التي حظيت بتقدير بالغ قديما وحديثا مـن كلّ المتلقيّن لما وهي رواية "دي باوه لندوعان كعبة".

الكلمات المفتاحية: ترجمة، رواية، مشكلة، حل

\begin{abstract}
Translation is a tool of transferring science and knowledge from and to different languages to exchange culture and civilization, by translation we know other cultures and their arts and literatures. This research seeks to identify some problems of translation of literary texts, especially the novel from Indonesian to Arabic, and aims to find solutions to these problems. The researcher chose to translate one of the works of literary literature, which has received a great appreciation from all recipients of the novel, "Di Bawah Lindungan Ka'bah."
\end{abstract}

Keywords: translation, novel, problem, solution

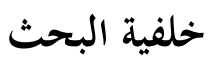

تقسم الترجمة في مختلف الأدبيات التي تتناول نظرية الترجمة إلى نوعين رئيسيين هما الترجمة الحرفية (literal translation) والترجمة الحرة (Free translation) ويذكر في بعض الدراسات نوع ثالث هو المحاكاة (imitation) وهي الترجمة التي تنطوي على درجة كبيرة من التصرف بحيث لا يبقى من النص الأصلي إلا فكرته الرئيسية. وبتري المقابلة بين هذه الأنواع 
وبين مزايا كل منها ومشاكله، وكثيرًا ما تخلص دراسات الترجمة إلى أنه لا مفر من استخدام مزيج من نوعين لإنتاج ترجمة تفي بالغرض الأساسي وهو نقل النص من لغة إلى أخرى بأقل قدر من الخسارة، سواء في المعنى أم في الشكل. (1) 1. الترجمة الحرفية

يُسيء كثير من الباحثين إلى هذا النوع ويذكروها غالبا على أها ترجمة معيبة، ويخلطون بينها وبين الترجمة اللفظية، ويرون أها مطابقة كل المطابقة لطريقة حنين بن إسحاق التي ذكرها الصفدي حين قال: "أن ينظر (أي الناقل) إلى كل كلمة مفردة من الكلمات اليونانية وما تدل عليه من المعنى، فيأتي بلفظة مفردة من الكلمات العربية ترادفها في الدلالة

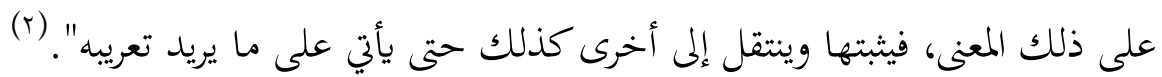

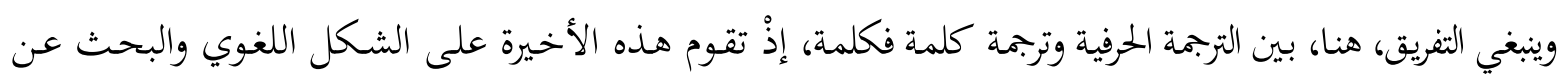
المقابلات في اللغة الهدف دون مراعاة لسياق النص ثم صياغة الجملة المترجمة وفقاً لتركيبتها في اللغة المصدر. أمـا الترجمة الحرفية فهي، وإن كانت تبحثث كذلك عن مقابل المفردات في اللغة المدف دون مراعاة للسياق، إلا أهـا تسعى، قدر المستطاع، أن تكون التركيبة اللغوية للغة المصدر مماثلة لنظيرتحا في اللغة الهدف. (r) يوضّح بيتر نيورمارك هذه الاختلافات المنهجية بين الترجمين حيث يقول عن ترجمة كلمة فكلمة : " توضع مفردات اللغة الهدف تحت مفردات اللغة المصدر مباشرة مع المحافظة على التركيبة اللغوية للغة المصدر. تُترجم كل كلمة بمفردها وتُعطى أكثر المعاني شيوعاً دون مراعاة للسياق. وتُترجم المفردات الثقافية فيها حرفيا " . أما عن الترجمة الحرفية، فيقول : " تتحول التراكيب اللغوية للغة المصدر إلى أقرب تراكيب لغوية ممكنة تقابلها في اللغة المدف، لكن تُترجم الكلمات

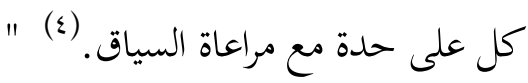
انظر إلى النص التالي، ستتمّ ترجمته على الطريقتين اللفظية والحرفية ولاحظ الفرق بينهما:

Day by day I float my paper boats one by one down

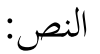

The running stream.

In big black letters I write my name on them and the name of the village where I live.

I hope that someone in some strange land

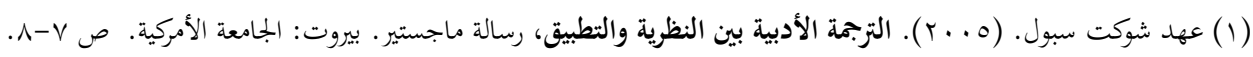

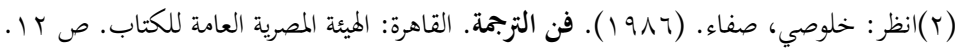

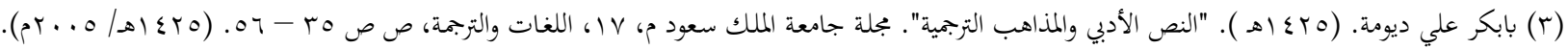
الرياض: كلية اللغات والترجمة جامعة الملك سعود. ص 9 ه.

Newmark, Pieter. (1988). A textbook of Translation. New York - London: Prentice Hall International. pp. 45 - ( $\varepsilon)$ 
${ }^{(\circ)}$ will find them and know who I am.

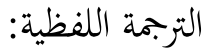

$$
\begin{aligned}
& \text { يوما بعد يوم، أنا أعوّم ورقتي قوارب واحدة تلو الأخرى إلى أسفل } \\
& \text { تيار تشغيل. } \\
& \text { في رسائل سوداء كبيرة وأنا أكتب اسمي عليها و لئو } \\
& \text { اسم القرية التي أعيش فيها. } \\
& \text { وآمل أن شخصا ما في بعض أرض غريبة } \\
& \text { سوف يجدها ويعرف من أنا }
\end{aligned}
$$

$$
\begin{aligned}
& \text { كل يوم } \\
& \text { أعوّم زوارقي الورقية } \\
& \text { واحدة بعد أخرى رولي الخرسي } \\
& \text { في مجرى النهر } \\
& \text { وأكتب فوقها اسمي } \\
& \text { واسم قريتي } \\
& \text { بأحرف سوداء كبيرة } \\
& \text { والأمل يحدولي بأن يعثر عليها } \\
& \text { بعض الناس } \\
& \text { في بعض البلدان الغريبة }
\end{aligned}
$$

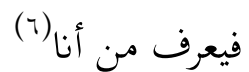

وواضح من من النص الأول أن الترجمة اللفظية رديئة لأها تستبدل اللفظ في لغة المصدر بلفظ في لغة الهدف دون النظر إلى السياق ولا إلى تراكيب اللغة المنقول إليها، فتصاب الترجمة بالركاكة والعجمة والخطأ والغموض. أما النص الثاني على

Tagore, Rabindranath. (2002). The Crescent Moon. New Delhi: Rupa \& Co. p. 5.(0)

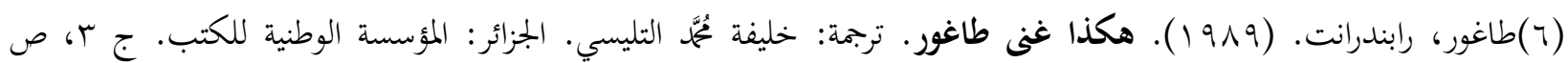

$$
. Y \mid Q-Y \backslash V
$$


طريقة الترجمة الحرفية فيحرص فيها المترجم على نقل معنى النص الأصلي وشكله، ويلتزم بالدقة والأمانة التزاما حرفيا (أي

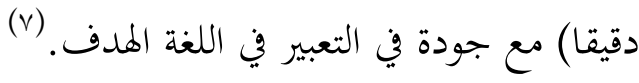
Y ب الترجمة الحرّة لعلنا نجد أفضل وصف للترجمة الحرة عند جورج شتاينر وهو منْ أهم من تناول الترجمة الحرة والنظرية التفسيرية

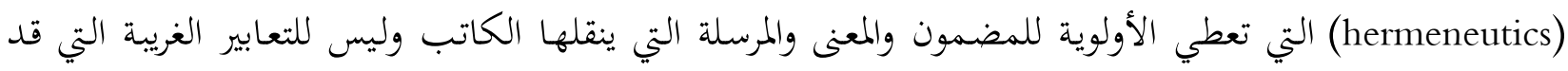
يتضمنها النص المترجم.

ويصف شتاينر عملية الترجمة بأها فهم النص وتفسيره باتباع الحركة التفسيرية (hermeneutic motion) التي

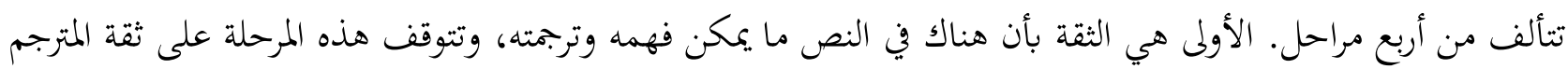

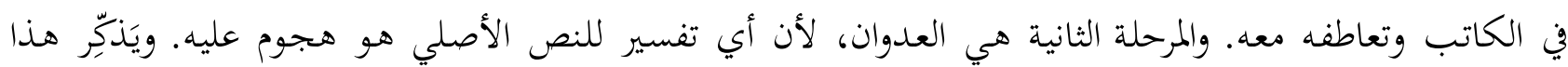

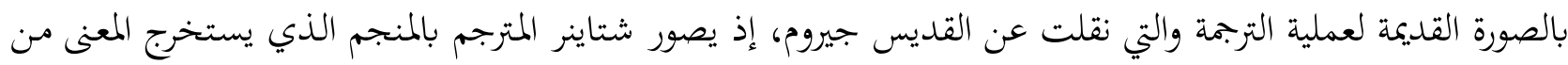

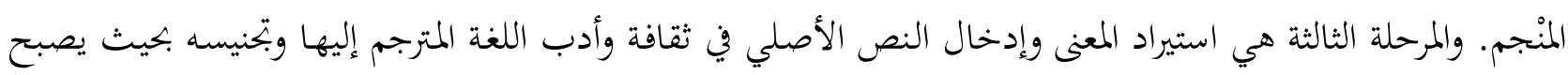

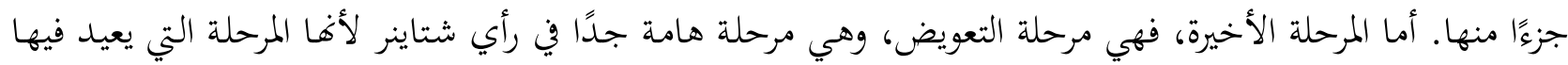

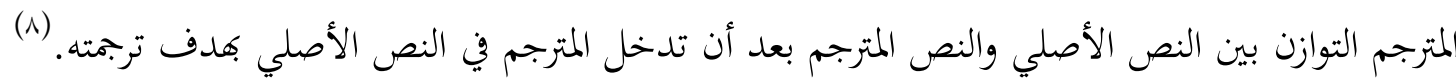

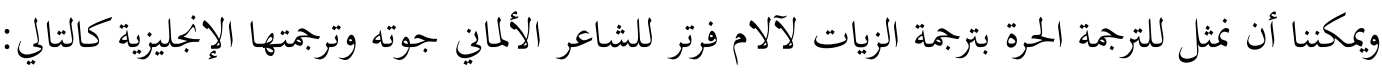

"How happy I am that I am gone! My dear friend, what a thing is the heart of man! To leave you, from whom I have been inseparable, whom I love so dearly, and yet to feel happy. I know you will forgive me".

$$
\text { وقد ترجم الزيات هذه العبارة كما يأتي: }
$$

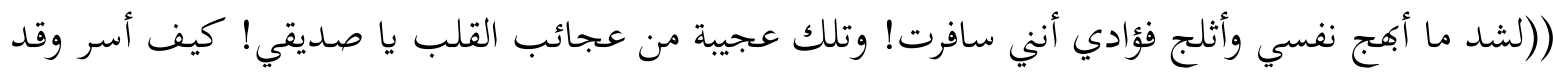

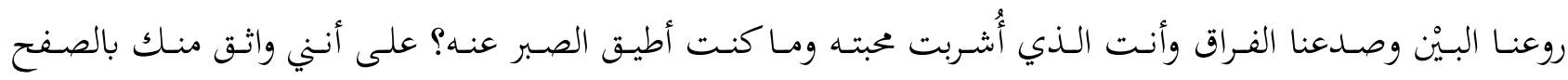
(9). (والمغفرة). (9)

\section{r. المزج بين التزجمة الحرفية والتزبمة الحرة}

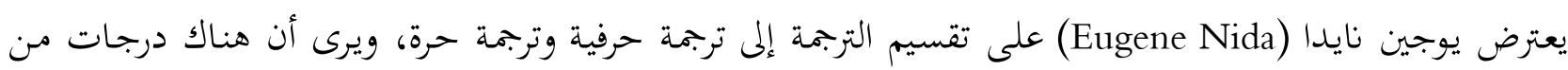

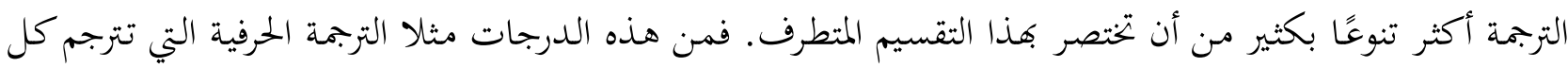

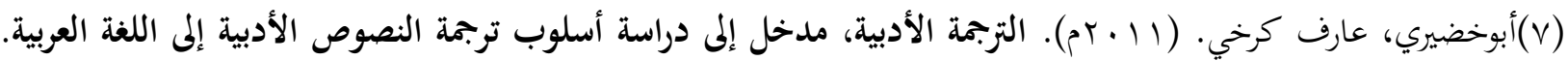

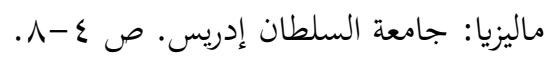

George Steine. (2000). The Hermeneutic Motion, In: Lawrence Venuti The Translation Studies. London and $(\wedge)$ New York: Routledge. pp. 186-191.

(9) خورشيد، إبراهيم زكي. (919 1). الترجمة ومشكلاتما. القاهرة: الهيئة المصرية العامة للكتب. ص.0.

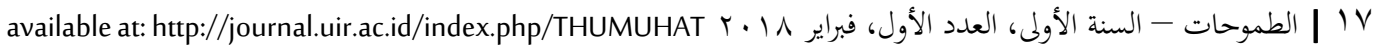


كلمة بكلمة وكل سطر بسطر، وقد سبق أن أشرنا إليها؛ والترجمة القريبة جدًا من المعنى اللغوي والشكل والتي تزود عادة بالحواشي المستفيضة التي تشرح التعابير والتراكيب الغامضة؛ والترجمة التي تمدف إلى إثارة الأثر نفسه عند القارئ وكأنه يقرأ النص الأصلي بغض النظر عن المضمون، وغير ذلك من أنواع الترجمة التي تتوقف على درجة الالنزام بحرفية النص أو

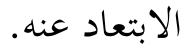

ويرى نايدا أن الاختلاف في أنواع الترجمة يعود إلى ثلاثة عوامل رئيسة هي طبيعة المرسلة التي ينبغي نقلها من لغة إلى أخرى؛ والغرض الذي يرمي إليه الكاتب وبالتالي المترجم؛ والجمهور الذي توجه إليه هذه المرسلة. والاختلاف الأساسي هو في تحديد الأولوية في المرسلة، هل هي للشكل أو للمضمون؟ فالشعر مثلا يعطي أهمية كبرى للشكل وأثره الجمالي، وهذا لا يعني أن المضمون غير هام، ولكن ينبغي نقله في شكل يحافظ على هذا الأثر الجمالي. أما النصوص الإرشادية، فمضموها أهم من الشكل. ولا بد من ترجمتها بنص مفهوم تمامًا لا مجال للالتباس فيه. وقد يتطلب هذا النوع من النصوص درجة كبيرة من التطويع والتحرر من القيود اللغوية في الترجمة. وأخيرًا، يجب أن تراعي الترجمة الجمهور الذي تتجه إليه لأن هذا يتحكم باختيار مستوى اللغة. فالقراء يختلفون في قدرة كل منهم على تفكيك الرموز كما يختلفون في بجالات اهتمامهم. (1.) ويخلص نايدا إلى أن هناك ابحاهين عامين في الترجمة وليس نوعان من الترجمة. الأول ابتحاه نحو المكافئ الشكلي formal) gequivalence) تغريب النص. وينصح نايدا بتزويد هذه الترجمات بالكثير من الحواشي لتوضيح مواطن الغموض. والاتجاه الآخر هو نحو المكافئ الدينامي (dynamic equivalence) ويهدف إلى الخروج بنص مطابق في جميع تفاصيله للخصائص اللغوية للغة التي يترجم إليها ولثقافة هذه اللغة. وهو ما يصفه شلاير ماخر بالتجنيس، وما يمكن وصفه أيضًا بالترجمة الحرة. إن الغرض من الترجمة هو الأساس الذي يحدد الخيارات المتاحة للمترجم. هذا ما جاء به هانس فيرمير (Hans Vermeer) في نظرية الترجمة الوظيفية أو نظرية الغرض. فنوع الترجمة التي سيتبعها المترجم يتوقف أولا وآخرًا على الغرض skopos) من ترجمتهـ ويقول فيرمير إن كل ترجمة يجب أن يكون لها هدف، وهو ما يشير إليه بالعبارة اليونانية skoposy)

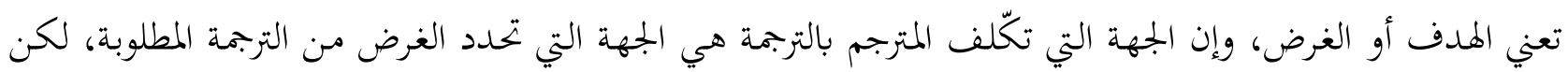
المترجم هو الذي يحدد الأسلوب الذي سيتبعه للإيفاء ههذا الغرض بصفته الشخص المختص والخبير بعملية الترجمة. فله أن يختار أي درجة من درجات التمسك بالنص الأصلي أو الابتعاد عنه وففًا لما يراه مناسبًا. (11)

Nida, Eugene. (1964). Toward a Science of Translating. Netherlands: Leiden. pp. 156-158.( • •)

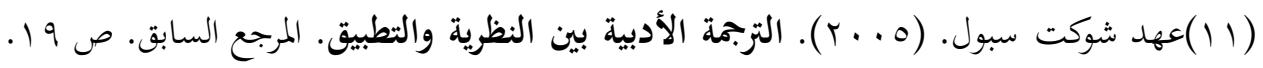

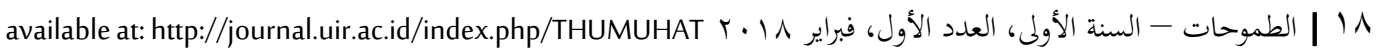




\section{تعريف بالرواية}

سمّى حمكا روايته هـذه بـ "دي باوه لندوعن كعبـة" وهي الرواية الثالثة مـن روايات حمكا، كُتبـت الرواية سـنة

جبا9 ا م في مدينة ميدان بسومطرة الشمالية، وذلك عندما كان حمكا رئيس تحرير مجلة فيدومان مشاركت ( Pedoman (.Masyarakat) هذا، ولاقت الرواية شهرة واسعة في جميع الأقطار الإندونيسية وماليزيا وسنغافورة وبروناي دار السلام وتمافت الناس من كل الأعمار والأجناس على قراءقها. وتمتعت هذه الرواية بمكانة مرموقة لدى الشعب الإندونيسي والملايوي عموما لما يجـدون فيها مـن بهجة التلقي وعذوبـة التنـاول ومـا تضمنته مـن صـدى الحياة الإنسـانية المعبرة والمعاني الأخلاقيـة المثالية والرسالة الدينية الهادفة، فهي ليست مجرد كتاب للتسلية يُستمتع به وكفى، فقد هدف حمكا من كتابتها إلى أن يقدم لقرائه نموذجا للرواية الإسلامية. ومن ثم قبلوها قبولا حسنا وأحبّوها حبا حتى إنه ما أحد عاث في زمافا إلا وعرفها، وما من مكتبة إلا والرواية في مقدمات كتبها. وتتكوّن الرواية من أربعة عشر فصلا وهي:

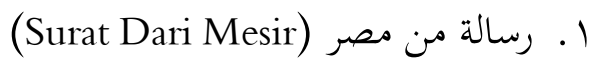

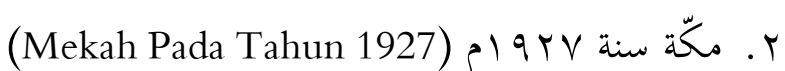
r. (Anak Yang Kematian Ayah) ع. المساعد (Penolong)

ه. بَّ يسمى هذا (Apakah Namanya Ini) 7. الحظ نفسه (Seperuntungan) V الثبوت والاغيار (Tegak Dan Runtuh) م. (Berjalan Jauh) (السفر) 9. خبر من القرية (Berita Dari Kampung) (Harapan Dalam Penghidupan) رجاء في الحياة 11 ( 1 ( الرسائل (Surat-Surat) r ا. تحت أستار الكعبة (Di bawah Lindungan Ka'bah) ب 1 ـ رسالة روسنة اللاحقة (Surat Rosnah Yang Menyusul Surat Telegram) 


\section{نوع الترجمة التي اخترتما لتزبمة الرواية}

ولتربمة رواية دي باوه لندوعان كعبة فضّلت النوع الثالث من الترجمة وهو المزج بين الترجمة الحرفية والترجمة الحرة

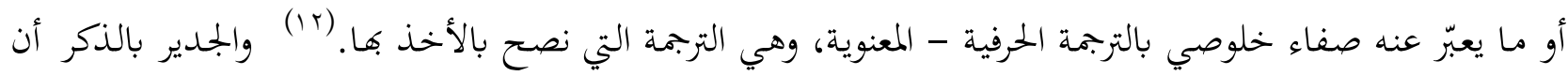

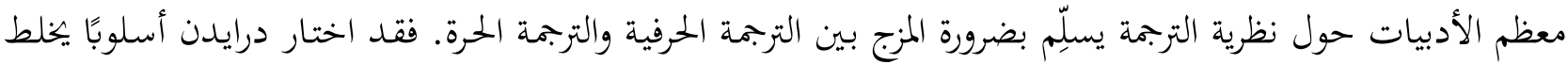

I thought to fit steer between the two extremes of paraphrase and literal translation;..."... بينهم بحيث يثير فضول القارئ بفعل غرابة النص نتيجة للترجمة الحرفية ويوضح له في الوقت ذاته فحوى النص المترجم بواسطة

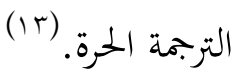

وفي رأيي أن الأخذذ بهذا النوع من الترجمة سيحقّق غرضي لترجمة هذه الرواية وهو نقل تجربة أدبية ثرية وإنتاج

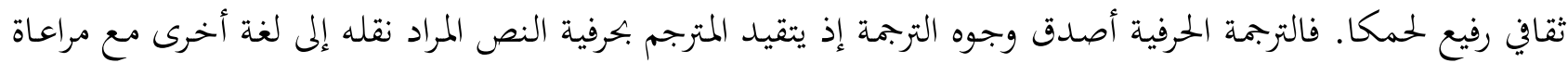

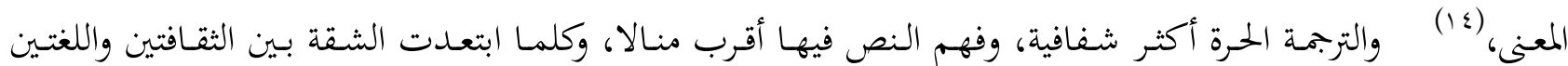

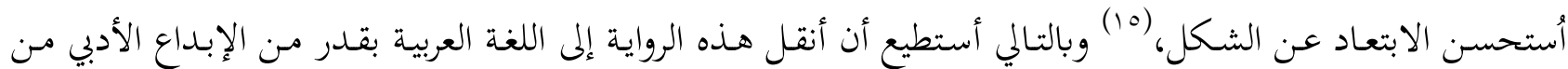
جانب، وأحافظ على خصائص حمكا وميزاته من جانب آخر. وعلى هذا فإني أتفق مع الزيات حيث يرى إن عمل المترجم الأدبي يتمثل في جهدين أسماسيين: أولمما جهده في

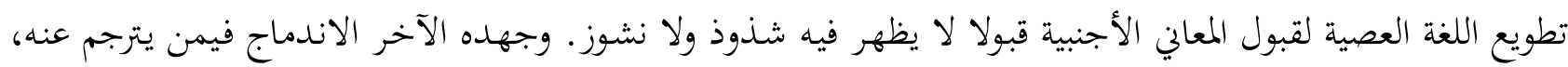
فيشعر بقلبه، وينظر بعينه، وينطق بلسانه. (1 (1) فالجهد الأول لغوي خـالص، والثاني فنّي صرف، وهو تقمص شخصية المؤلف "فيشعر بقلبه، وينظر بعينه،

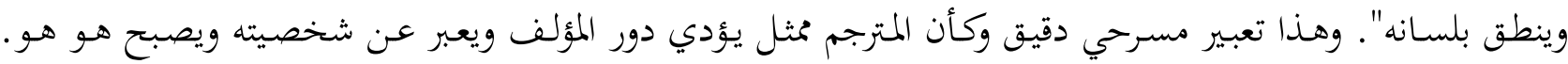
ويتضمن هذا التقمص عنصرين: الأول نقل شخصية الكاتب، وروحه ومشاعره، والثاني نقل أسلوبه، وطريقته، ومذهبه

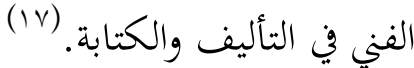

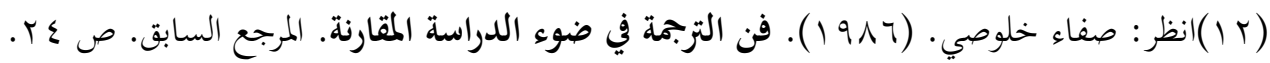

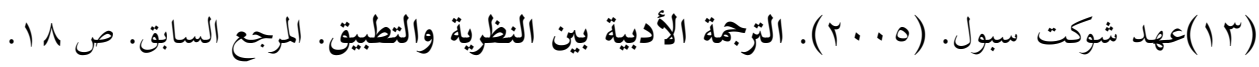

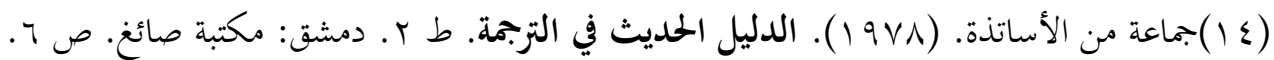

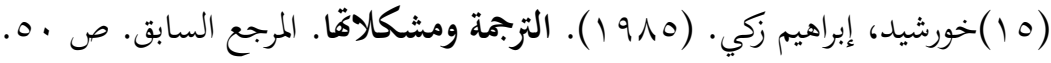

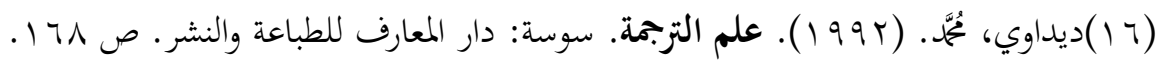

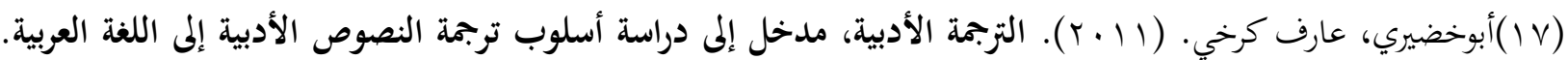

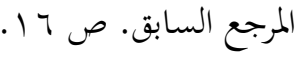

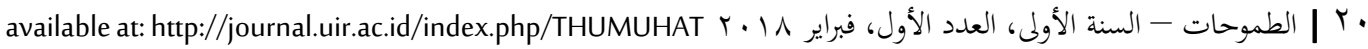


وكذلك أتفق مع رأي صفاء خلوصي حيث يقول: "أما نحن فنأخذ برأي وسط بين الرأيين فلا نحسن الأصل

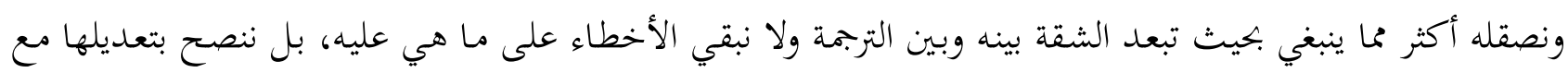

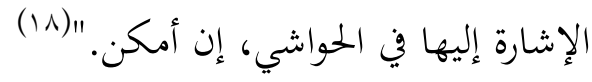

مشكلات تربمة الرواية

حينما شرعت في عملية الترجمة وتكمن الصعوبات والمشاكل في الأشياء التالية:

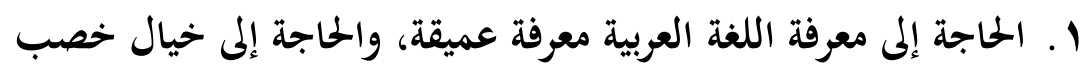

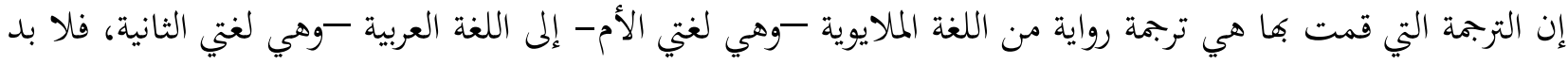

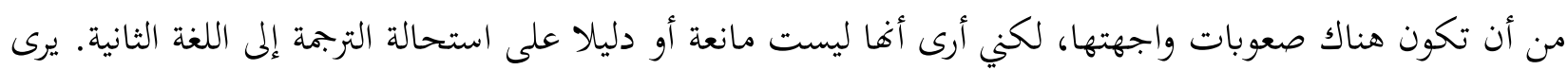

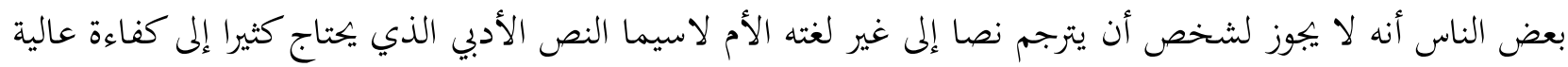

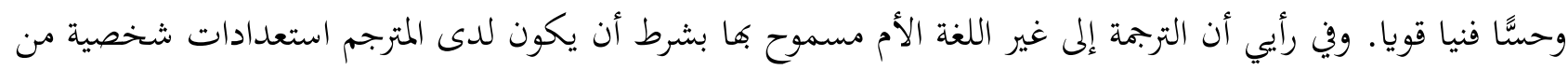

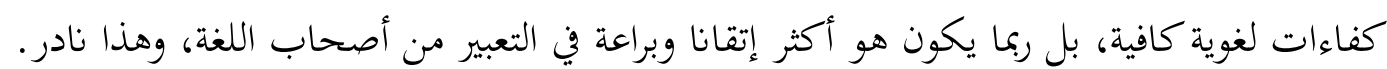

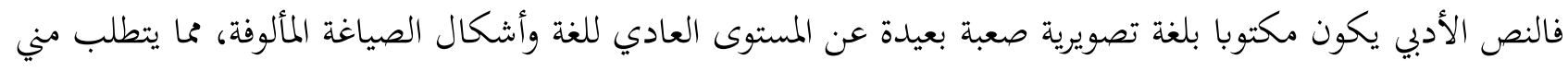

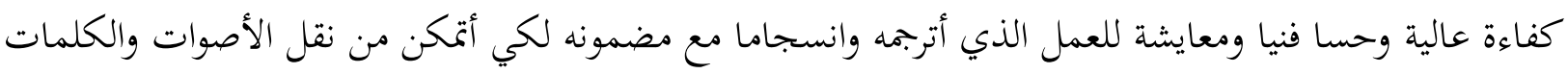

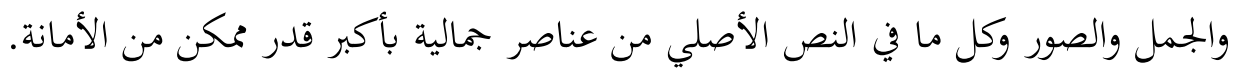

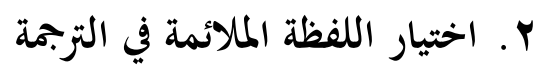

لا أجد صعوبة في فهم معاني الألفاظ الملايوبة، فالصعوبة تكمن في اختيار الألفاظ العربية الملائمة المناسبة لمعنى اللفظ

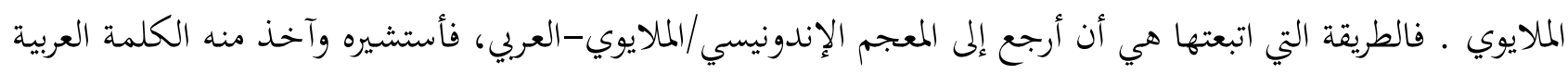

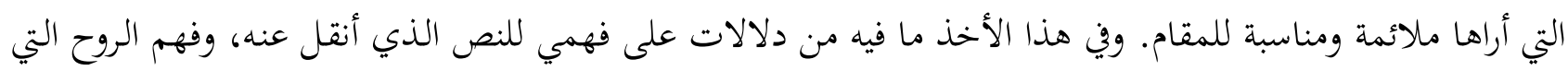

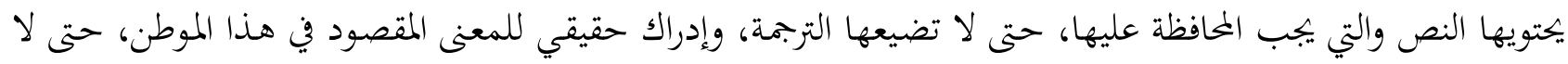

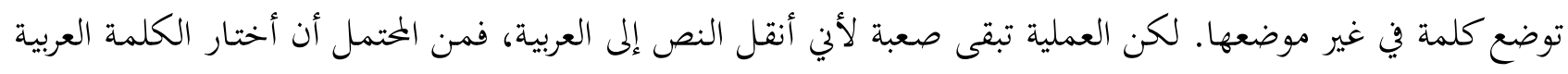

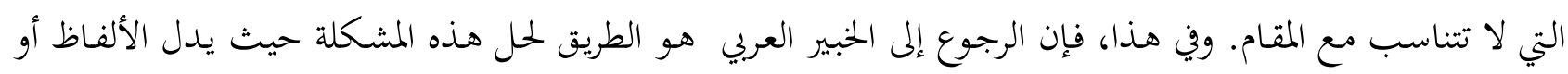

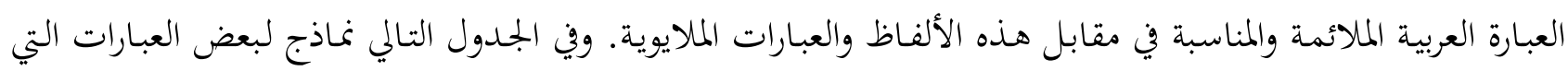

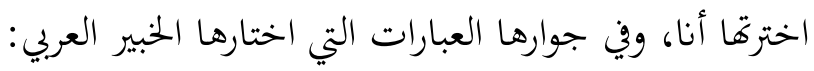

\begin{tabular}{|c|c|c|c|}
\hline العبارات التي اختارها الخبير & العبارات التي اخترتما & النص الملايوي & الرقم \\
\hline لقد تسلّمت الرسالة & لقد استلمت الرسالة & $\begin{array}{l}\text { Sudah saya terima } \\
\text { surat sahabat }\end{array}$ & 1 \\
\hline
\end{tabular}

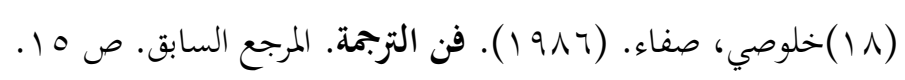




\begin{tabular}{|c|c|c|c|}
\hline الولايات المهزومة & الولايات المغلوبة & $\begin{array}{l}\text { Daerah-daerah } \\
\text { taklukannya }\end{array}$ & $Y$ \\
\hline 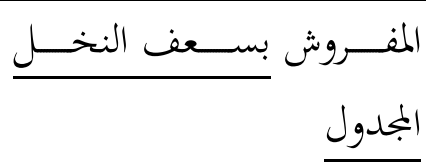 & المفروش بأوراق النخلة المطوية & $\begin{array}{l}\text { Berhamparkan daun } \\
\text { korma berjalin }\end{array}$ & $\mu$ \\
\hline
\end{tabular}

r. الاختلاف الثقافي أو البيئي

يـرتبط هـذا الموضـوع بمشـكلة عـدم قابليـة ترجمـة (untranslatability) تلك الكلمـات مـن اللغـة المصـدر (SL) إلى اللغـة المنقول إليها (TL) (19) وعلى سبيل المثال، ففي الثقافة الملايوية هناك مفهوم abang, adik، وهي مفاهيم غير موجودة godok perut ayam, rakit udang, bakso, في الثقافة العببية. كذلك تتواجد في البيئة الملايوية بعض الأكلات، مثل spempek, وبعض الملبوسات، مثل selendang, sarung, songkok والتي لا تتواجد في البيئة العربية. والحل الذي آخذه هو أن ألجأ إلى أسلوب transliteration، أي كتابة الكلمة في اللغة العربية حسب طريقة نطقها في اللغة الملايوية وإعطاء تفسير لهذه الكلمة بين قوسين أو في هامش الصفحة.

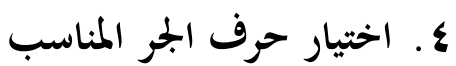

إن لحروف الجر فائدة كبيرة في ربط كلمتين أو جملتين بينهما علاقة مكان أو زمان أو علّة أو غاية أو اتحاد أو انفصال أو ضدّية. لكن باعتبارها أحرف كثيرة وذات معالٍ عديدة فإها كثيرا ما تشكل صعوبة في اختيارها ووضعها في مكاها المناسب. وقد واجهت هذه المشكلة في ترجمة هذه الرواية أذكر هنا بعض الأمثلة:

\begin{tabular}{|c|c|}
\hline الصحيح & الخطأ \\
\hline موافق عليه & موافق به \\
\hline يضحكون منيّ & يضحكون عليّ \\
\hline وصلت إلى مكة & وصلت في مكة \\
\hline
\end{tabular}

وكان سبب هذا الخطأ في رأيي تأثير لغة الأم لدى المترجم، حيث يبحث عن حرف الجر الذي يتصور في ذهنه أنه يوافق معنى الكلمة في اللغة الأصل. أما الخطوة التي اتخذها لحل هذه المشكلة أو للتقليل من الوقوع في الخطأ فيها فهي تكمن في الاطلاع أكثر على معاني هذه الأحرف والقراءة الكثيرة للنصوص العربية التي ترد فيها هذه الأحرف في استعمالاتحا الصحيحة.

\section{ه. ت ترجمة الصور البيانية}

مما لا شـكّ فيه أن الصور البيانية في أية لغة من اللغات تُعدّ صورة صادقة للنـاطقين بها، إذ يتجلى فيها تراثهم العلمي والأدبي والشعبي، فهي تعبّر عن تصوراتم وبحاربهم في الحياة. ويرى بيتر نيومارك Peter Newmark) أن الهدف الرئيس من استخدام الصور البيانية هو وصف الشيء أو الحـدث أو الصفة بطريقة أشمل وأوجز وأكثر تعقيداً مما هو متاح لنا

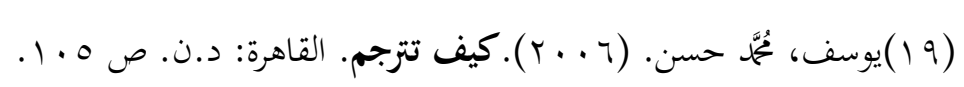


باستعمال اللغة العادية.(·) مـن هنا كانت الصور البيانية عنصراً مـن عناصر القوة والتأثير؛ لذلك نجد أن أهل اللغة

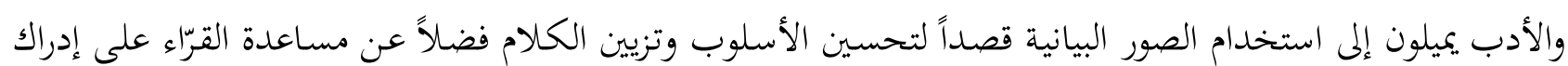

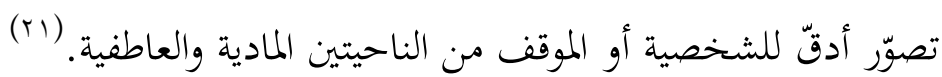
والصور البيانية في اللغة الملايوية تنقسم إلى قسمين أساسيين:

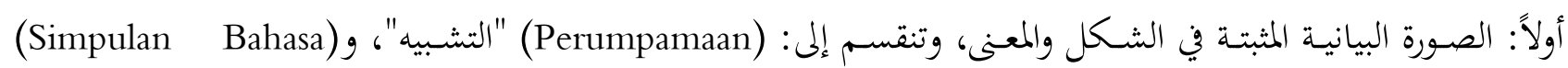

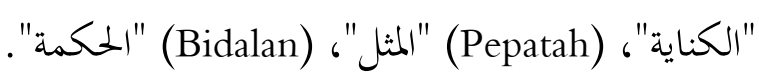

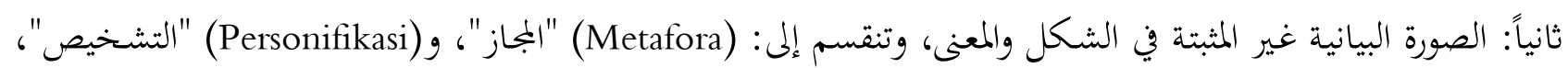

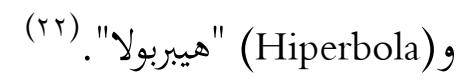

لقد اختلف العلماء حول إمكانية ترجمة الصور البيانية لخّصها أوليف كلاسي (Olive Classe) في قوله: فبعض

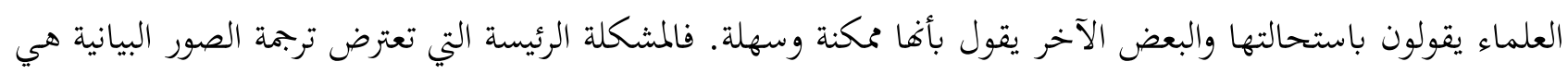

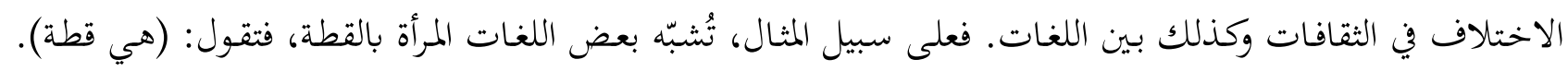

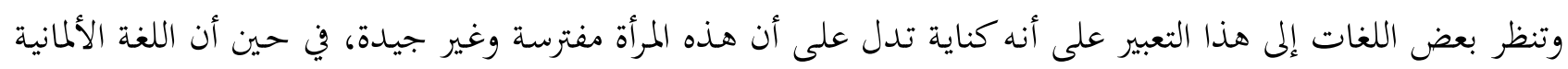

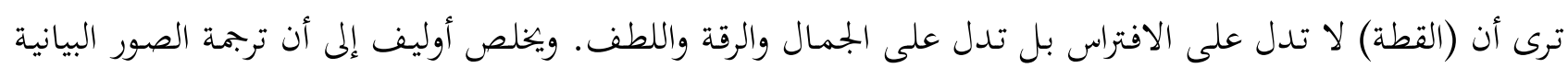

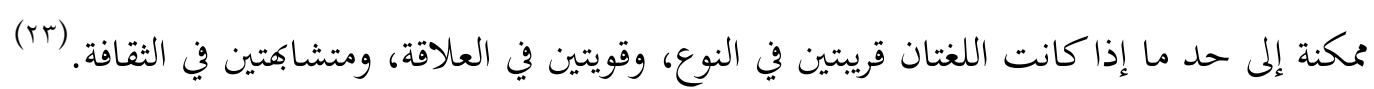
وللتخلّص من مشكلة ترجمة الصور البيانية اتبعت الأساليب التالية:

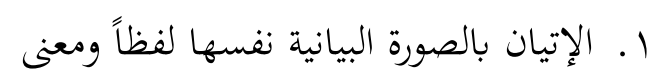

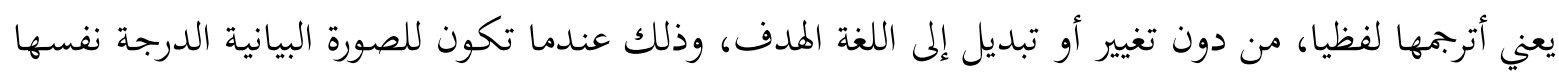

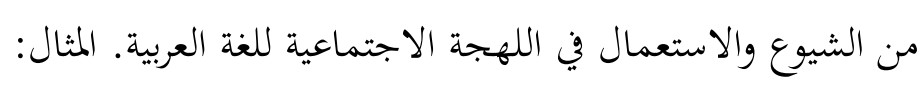
Awan gelap yang menutup keningnya hilanglah dari sedikit ke sedikit...." ( HAMKA, DBLK: 9) الترجمة: (20) السحب السوداء التي كانت تغطي وجهه بدأت تغيب قليلا قليلا...

$$
\text { ومن أمثلة تربمة التشبيه بالتشبيه نفسه في لغة المدف (العربية): }
$$

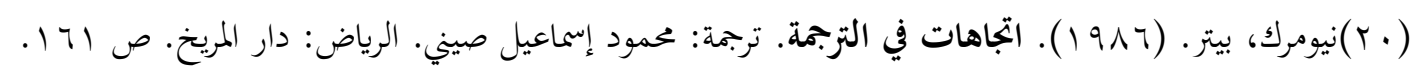

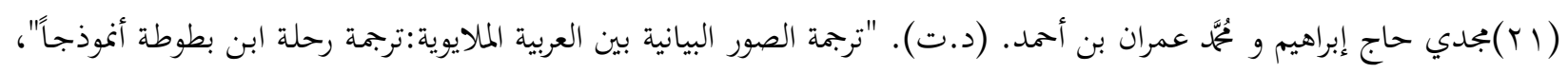

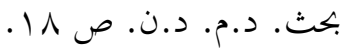

$$
\begin{aligned}
& \text { r. }
\end{aligned}
$$

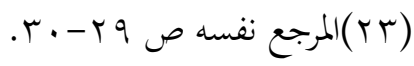

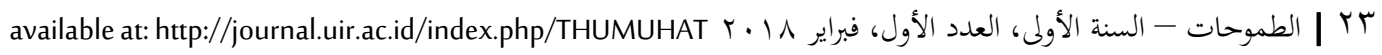


Anakanda tahu bahawa jika anakanda mencurahkan cinta kepadanya, takkan ubahnya seperti seorang yang mencurahkan semangkuk air tawar ke dalam lautan yang amat luas; laut tak ) \akan berubah sifatnya kerana semangkuk air itu...( HAMKA, DBLK:

الترجمة:

"وأنا أعلم لو أني أحبتها فحالي لا تكون إلا مثل من يصبّ كوبا من ماء عذب في البحر الفسيح، فلن يغير شيئا من صفاته.... r. تحويل الصورة البيانية إلى مضموفها تتم عملية تحويل الصورة البيانية إلى مضمونها في هذا الأسلوب مـن خلال تحليل المضمون وتفكيك مكوناته. فقد لجأت كثيرا إلى هذا الأسلوب في ترجمة الصور البيانية لهذه الرواية.

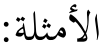

1. "Kedua ialah untuk menjadi cermin perbandingan orang-orang yang hidup kemudian daripada mereka..." ( HAMKA, DBLK: r)

وليكون عبرة لمن يعيش بعدهما ثانيا

لو ترجنا "cermin perbandingan" إلى العربية بصورتها الأصلية فهي تعني "مِرآة مقارنة"، والكناية بهذه الصيغة غير معروفة عند العرب، فلا يمكن ترجمتها إلى العربية ترجمة حرفية. لذلك يتوجب علينا أن نحلل مدلول الكناية الملايوية أولا، ثم ننقل مضموفا، فنقول: (العبرة).

2. "Betapa besar hati saya ketika melihat ka'bah.. "( HAMKA, DBLK: 4)

فالمجاز "Besar hati"(كبير القلب) لا يمكن ترجمته إلى العربية ترجمة حرفية، فنقلت مضمونه وهو يعني الفرح الشديد. 3. "Hampir mamak terlalai dari janji kita. Tadi mamak pergi ke rumah orang sebelah.". (

HAMKA, DBLK: 30) 
كدتُ أنسى وعدنا. ذهبت إلى بيت الجيران قبل قليل

"orang sebelah"

\section{الحخلاصة}

إن الترجمة من اللغة الإندونيسية -وهي لغتي الأم- إلى اللغة العربية -وهي لغتي الثانية تكمن صعوبات ومشكلات، ومن هذه المشكلات بالتطبيق على رواية "دي باوه لندوعان كعبة" هي: الحاجة إلى معرفة اللغة العربية معرفة عميقة، والحاجة إلى خيال خصب، ومشكلة اختيار اللفظة الملائمة في الترجمة، ومشكلة الاختلاف الثقافي أو البيئي، ومشكلة اختيار حرف الجر المناسب، ومشكلة ترجمة الصور البيانية. أما طرق التخلص عن هذه المشكلات منها: تكوين استعدادات شخصية من كفاءات لغوية، وحس فني ومعايشة للعمل الذي ترجم، والرجوع إلى المعجم الإندونيسي/الملايوي-العربي، والاستفسار من الحنبير العربي، واللجوء إلى أسلوب transliteration، أي كتابة الكلمة في اللغة العربية حسب طريقة نطقها في اللغة الملايوية وإعطاء تفسير لهذه الكلمة بين قوسين أو في هامش الصفحة، وكثرة الاطلاع على معاني أحرف الجر والقراءة الكثيرة للنصوص العربية التي ترد فيها هذه الأحرف، والتخلّص من مشكلة ترجمة الصور البيانية بالتباع أساليب الإتيان بالصورة البيانية نفسها لفظاً ومعنى وتحويل الصورة البيانية إلى مضموها.

\section{المراجع}

أبوخضيري، عارف كرخي. (1 (1). الترجمة الأدبية: مدخل إلى دراسة أسلوب ترجمة النصوص الأدبية إلى اللغة العربية. ماليزيا: جامعة سلطان إدريس. أبوخضيري، عارف كرخي. (11 ( + ). الترجمة الأدبية، مدخل إلى دراسة أسلوب ترجمة النصوص الأدبية إلى اللغة العربية. بابكر علي ديومة. (0 إك اهـ ). "النص الأدبي والمذاهب الترجمية". مجلة جامعة الملك سعود م، VI V، اللغات والترجمة، الرياض: كلية اللغات والترجمة جامعة الملك سعود.

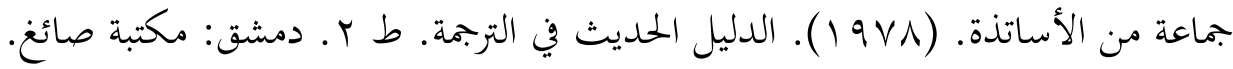
خلوصي، صفاء. (1919 1). فن الترجمة. القاهرة: الهيئة المصرية العامة للكتاب. خورشيد، إبراهيم زكي. (910 (1). الترجمة ومشكلاتقا. القاهرة: الهيئة المصرية العامة للكتب. ديداوي، عُمَّه. ( ( 99 1). علم الترجمة. سوسة: دار المعارف للطباعة والنشر.

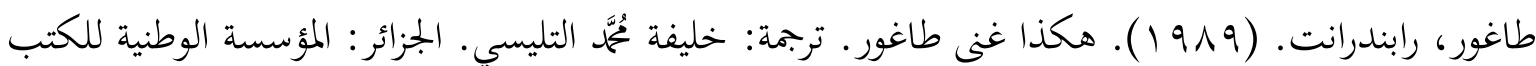




$$
\begin{aligned}
& \text { عهد شوكت سبول. (0 . . ץ). الترجمة الأدبية بين النظرية والتطبيق، رسالة ماجستير. بيروت: الجامعة الأمريكية. } \\
& \text { عهد شوكت سبول. (0 . ․ ( ). الترجمة الأدبية بين النظرية والتطبيق، رسالة ماجستير. بيروت: الجامعة الأمركية } \\
& \text { مجدي حاج إبراهيم و مُحمَّ عمران بن أحمد. (د.ت). "ترجمة الصور البيانية بين العربية الملايوية:ترجمة رحلة ابن بطوطة }
\end{aligned}
$$

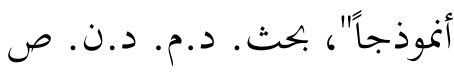

$$
\begin{aligned}
& \text { نيومرك، بيتر . (7 (9 1 ). ابتحاهات في الترجمة. ترجمة: محمود إسماعيل صيني. الرياض: دار المريخ. }
\end{aligned}
$$

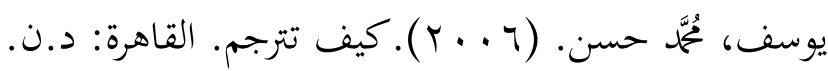

George Steine. (2000). The Hermeneutic Motion, In: Lawrence Venuti The Translation Studies. London and New York: Routledge.

Newmark, Pieter. (1988). A textbook of Translation. New York - London: Prentice Hall International.

Nida, Eugene. (1964). Toward a Science of Translating. Netherlands: Leiden.

S. Takdir Alisjahbana. (1977). Dari Perjuangan dan Pertumbuhan Bahasa Indonesia dan Bahasa Malaysia Sebagai Bahasa Moderen. Jakarta: PT. Dian Rakyat.

Tagore, Rabindranath. (2002). The Crescent Moon. New Delhi: Rupa \& Co. 